\title{
In-Plane and Through-Plane Gas Permeability of Carbon Fiber Electrode Backing Layers
}

\author{
Jeff T. Gostick, Michael W. Fowler*, Mark D. Pritzker, \\ Marios A. loannidis and Leya M. Behra \\ Department of Chemical Engineering \\ University of Waterloo, Waterloo, Ontario, Canada N2L 3G1
}

${ }^{*}$ Corresponding Author 


\section{Abstract}

The absolute gas permeability of several common gas diffusion layer (GDL) materials for polymer electrolyte membrane fuel cells was measured. Measurements were made in three perpendicular directions to investigate anisotropic properties. Most materials were found to display higher in-plane permeability than through-plane permeability. The permeability in the two perpendicular in-plane directions was found to display significant anisotropy. Materials with the most highly aligned fibers showed the highest anisotropy and the permeability could differ by as much as a factor of 2 . In-plane permeability was also measured as the GDL was compressed to different thicknesses. Typically, compression of a sample to half its initial thickness resulted in a decrease in permeability by an order of magnitude. Since the change in GDL thickness during compression can be converted to porosity, the relationship between measured permeability and porosity was compared to various models available in the literature, one of which allows the estimation of anisotropic tortuosity. The effect of inertia on fluid flow was also determined and found to vary inversely with permeability, in agreement with available correlations. The results of this work will be useful for 3D modeling studies where knowledge of permeability and effective diffusivity tensors is required. 


\section{Introduction}

The commercialization of the polymer electrolyte membrane fuel cell (PEMFC) is highly anticipated, but several technological difficulties must still be addressed. Some of the main challenges are to prolong membrane life, increase power density and reduce the platinum loading [1]. The last two points can be addressed by improving mass transfer rates in the porous electrodes and porous backing layers, or gas diffusion layers (GDLs). Higher mass transfer rates would allow higher current densities to be achieved and would also enable higher reactant concentrations to be maintained in the catalyst layer, thereby reducing the amount of catalyst required. If the mass transfer rates through the GDL are to be improved, then a more complete understanding of the transport properties of GDL materials is needed.

In this work, a detailed analysis of single-phase fluid flow through GDLs is presented with the aim of measuring the transport properties associated with convection, namely the permeability and the inertial coefficient. For sufficiently low fluid velocities, single-phase flow through a porous medium is described by Darcy's Law [2]:

$$
-\nabla P=\frac{\mu}{K} \vec{v}
$$

where $K$ is the absolute permeability of the porous material, $\mu$ is the viscosity of the flowing fluid, $\vec{v}$ is the superficial velocity of the fluid and $P$ is the pressure. In 
the creeping flow regime, viscous interactions between the fluid and the porous solid are the dominant source of pressure loss. At higher velocities, an inertial pressure loss is incurred by the acceleration and deceleration of the fluid as it flows along curved streamlines through the tortuous paths of the porous media. This phenomenon, termed the Forchheimer effect, manifests itself as a nonlinearity in the dependency of the flowrate on the pressure drop. Incorporating this effect into Darcy's law results in the Forchheimer equation [2]:

$$
-\nabla P=\frac{\mu}{K} \vec{v}+\beta \rho|\vec{v}| \vec{v}
$$

where $\rho$ is the fluid density and $\beta$ is the inertial coefficient. The inertial coefficient is also referred to as the Forchheimer coefficient or the non-Darcy coefficient.

Although diffusion from the flow channels to the catalyst layer is considered the primary mode of transport for delivery of reactants [3], convective transport is important in several instances. Forced convection in the plane of the GDL can occur since a pressure difference often exists between neighboring flow channels. It has been experimentally observed that over-land convection can significantly improve cell performance and delay onset of the mass transfer limiting regime [4]. This behavior has been the focus of several numerical studies [5-7]. Pharaoh [5] modeled flow along serpentine channels and calculated the fraction of gas that would flow over the land into the neighboring channel as a function of the GDL permeability. It was found that nearly all the gas would flow in the channel if the GDL permeability is below $10^{-12} \mathrm{~m}^{2}$, but over- 
land convection would become more important as the permeability increased. As much as $50 \%$ of the gas fed to a channel could be expected to flow by convection through a GDL with a permeability of $10^{-10} \mathrm{~m}^{2}$. The permeability of commercially available GDLs is typically in the middle of this range with values from 1 to $50 \times 10^{-12} \mathrm{~m}^{2}$. The interdigitated flow field [8] is designed to maximize the beneficial effects of in-plane convection by forcing all flow through the GDL. Significant performance improvement is achieved by this design, but it causes prohibitive pressure drops through the cell and so has not been widely employed. It has also been suggested by modeling studies [9] that a through-plane pressure gradient may exist between the flow channel and the catalyst layer which would lead to convective mass transfer. This pressure gradient is caused by the consumption of reactants at the catalyst layer and condensation of the product water. Cell assembly also plays an important role in these convective mass transfer processes since cell compression reduces the permeability. Lee et al. [10] studied the effect of cell compression and found that performance first improved but then deteriorated as the degree of compression was increased. They attributed this behavior to the competing effects of improved contact resistance and reduced GDL permeability as the cell was compressed more tightly. Since cell compression is an integral part of PEMFC assembly, it is important to understand how the compression of the GDL affects performance.

Very few experimental measurements of GDL permeability have been reported in the open literature. Williams et al. [11], Ihonen et al. [12] and Mueller et al. [13] 
measured the through-plane permeability of several materials. However, almost all the materials tested in these studies were coated with a microporous layer (MPL). This confounding factor makes it impossible to determine the transport properties of the GDL substrate material alone. Through-plane permeability was also measured by Prasanna et al. [14] for two types of materials with varying PTFE content and no MPL. Mathias et al. [3] measured through-plane permeability of a single sample with no MPL, but reported the result only as an approximate range of values. Ihonen et al. [12] measured in-plane permeability for each material as a function of compression. They found that the permeability decreased as the compression force applied to the GDL was increased. Dohle et al. [15] measured the in-plane permeability of a single type of GDL, but with different MPL properties. They reported the permeability as a function of GDL thickness instead of compression. Again, because of the presence of the MPL, neither of these studies revealed the intrinsic transport properties of the GDL. Mathias et al. [3] also measured in-plane permeability for a single sample, but again reported their results only as an approximate range. The above mentioned studies are also limited in that the effect of directional anisotropy on in-plane permeability was not investigated. Also, no attempt was made to correlate or predict GDL permeability using established theories of flow through fibrous porous media.

In the present study, permeability is measured in the through-plane and both inplane directions for a variety of GDL substrate materials without MPL. For the in- 
plane tests, the effect of directionality is investigated to determine the effects of fiber orientation and anisotropy. Measurement of the permeability of the samples in all three principal directions provides much more detailed information about GDL structure and transport behavior. Additionally, the in-plane permeability is measured as a function of GDL compressed thickness to determine the effect of cell assembly on GDL permeability. Since change in sample porosity during compression is also known, it is possible to study the relationship between permeability and porosity and to verify the applicability of known permeabilityporosity models. The inertial coefficient is also determined for all samples and compressions and its values are compared with previously developed correlations. 


\section{Materials and Methods}

\subsection{In-Plane Gas Permeability}

The in-plane gas permeability was measured as a function of GDL thickness to simulate conditions in an assembled cell, which is significantly compressed in order to promote good electrical contact between layers and ensure a tight gas seal. The experimental apparatus for these measurements is shown in Fig. 1. The sample size for this test was $63.2 \mathrm{~mm}$ wide by $20 \mathrm{~mm}$ long. The sample was compressed between two plates with the spacing controlled by placing feeler gauges of known thickness between them. Using a torque wrench, the plates were tightened by two bolts to a torque of about $20 \mathrm{~N} \cdot \mathrm{m}$ each. Tests were performed to confirm that the test cell was sufficiently rigid and that the results did not depend on the bolt torque (i.e. the test cell did not deform when tightened). A bolt torque of $20 \mathrm{~N} \cdot \mathrm{m}$ was found to be sufficient to compress all samples and so was maintained at this level throughout all experiments for consistency. It was also verified that the test cell presented negligible pressure drop in the absence of sample to ensure that all observed pressure loss could be attributed to the sample alone. The sides of the cell were sealed by clamping a face plate on each side. A rubber gasket between the face plates and the body of the cell provided the gas seal. Seals along the back edge of the header slots were created using silicone putty. This malleable material yielded as the spacing between the plates was reduced and provided a reliable seal. The seal was 
tested before each run by closing the outlet and pressurizing the system to 400 $\mathrm{kPa}$. The setup could hold pressure indefinitely after the air supply was stopped.

The flow rate was measured on the outlet side using a digital flow meter (Omega FVL-1604-A, +/-0.5\% FS). A pressure gauge (Setra 209, +/-0.25\% FS) monitored the inlet pressure and the outlet was taken as atmospheric pressure since the presence of the flow meter in the line presented negligible pressure drop. Measurements for at least 10 flow rates were obtained at each GDL thickness. The local barometric pressure was recorded since absolute pressure is required in the data analysis (Section 2.3).

\subsection{Through-Plane Gas Permeability}

Through-plane permeability was measured using the set-up shown in Fig. 2. In this arrangement, gas was fed through the sample at a fixed flow rate and the resultant pressure drop was measured. The sample was circular with a diameter of $25.4 \mathrm{~mm}$. The GDL was secured between the two plates and a gas-tight seal was easily obtained given the low gas pressures used during the experiment (<15 Pa). The differential pressure sensor (Omega PX653, accuracy $+/-0.1 \%$ FS) spanned a range of -0.05 to 0.05 inches of water column ( 15 Pa to $15 \mathrm{~Pa})$. The flow rate was measured at the outlet using a digital flow meter (Omega FVL$1604-\mathrm{A},+/-0.5 \% \mathrm{FS})$. The pressure drop was obtained for at least 10 flow rates for each sample. The local barometric pressure was also recorded. 


\subsection{Data Analysis}

Solution of Darcy's law for the one-dimensional flow of a compressible fluid results in the following equation [16]:

$$
\frac{\left(P_{I N}^{2}-P_{O U T}^{2}\right)}{2 L R T / M W_{A I R}}=\frac{\mu}{K} m^{\prime}
$$

where $P_{\text {IN }}$ is the inlet pressure, $P_{\text {OUt }}$ is the outlet pressure, $L$ is the length of the sample, $R$ is the universal gas constant, $T$ is the temperature, $M W_{\text {AIR }}$ is the molecular weight of air and $m^{\prime}$ is the mass flux through the sample. Eq.(3) is valid when the gas velocity is small and viscous drag is the dominant cause of

pressure loss. At high velocity, inertial pressure losses become significant and Darcy's law must be modified to account for this effect. For a compressible fluid, solution of modified Darcy's law, or Forchheimer equation, leads to [16]:

$$
\frac{\left(P_{I N}^{2}-P_{\text {OUT }}^{2}\right)}{2 L R T / M W_{A I R}}=\frac{\mu}{K}\left(m^{\prime}\right)+\beta\left(m^{\prime}\right)^{2}
$$

At low velocities, the second term on the right hand side vanishes and Darcy's law is recovered. The permeability and inertial coefficient can be obtained by fitting Eq.(4) to experimental data and extracting the linear and quadratic coefficients, respectively. The viscosity of air was taken to be $1.85 \times 10^{-5} \mathrm{~Pa} \cdot \mathrm{s}$ for all runs and assumed to be independent of gas pressure [17]. 


\subsubsection{GDL Compression and Porosity Conversion}

During this work, the permeability was measured as a function of GDL compressed thickness. In order to compare experimental results with permeability models, it is necessary to convert the change in GDL thickness to a change in porosity. To this end, it is assumed that the fibers of the GDL are incompressible and all the reduction in bulk volume during compression arises from the reduction of pore volume. If it is additionally assumed that the sample deforms only in the direction of compression, then the porosity of the compressed sample may be determined from its compressed thickness as follows:

$$
V_{p, C}=V_{b, C}-V_{s}=V_{b, C}-\left(1-\varepsilon_{o}\right) V_{b, o}
$$

where $V_{\mathrm{p}, \mathrm{C}}$ is the pore volume of the compressed sample, $V_{\mathrm{b}, \mathrm{C}}$ is the bulk volume of the compressed sample and $V_{\mathrm{s}}$ is the solids volume, which is assumed to remain constant as the sample is compressed. $V_{\mathrm{b}, \mathrm{o}}$ and $\varepsilon_{0}$ are the bulk volume and porosity of the uncompressed sample, respectively. Eq.(5) can be rearranged to give the porosity of the compressed sample as:

$$
\varepsilon_{C}=\frac{V_{p, C}}{V_{b, C}}=1-\frac{\left(1-\varepsilon_{C}\right)}{V_{b, C} / V_{b, o}}
$$

\subsection{Materials}

Specific information for each of the GDL materials tested is listed in Table 1. A micrometer was used to verify thickness. Porosity values were taken from 
mercury intrusion porosimetry (MIP) measurements conducted using a Poremaster from Quantachrome Instruments (Boynton Beach, FL). Thickness and porosity values so measured were found to agree with available manufacturer specifications. Fiber diameter was estimated from SEM micrographs. Through-plane permeability was measured for three separate samples of each material. In-plane measurements were repeated at least twice (except in the case of Toray 090, due to limited materials). The in-plane permeability was tested in two perpendicular directions to determine if any anisotropy existed due to fiber orientation. All samples for both tests were taken from the same master sheet of material. 


\section{Results}

\subsection{In-Plane Permeability}

The in-plane permeability measurements showed a non-linearity due to the Forchheimer effect. Fig. 3 shows typical data obtained from these experiments. The permeability can be determined for each sample compression by fitting Eq.(4) to the data, yielding the results shown in Fig. 4a. The coefficient of correlation $\left(R^{2}\right)$ was 0.99 or higher for all runs. The compressed sample thickness is related to porosity $\varepsilon c$ using Eq.(6) to yield the results shown in Fig. 4b. As expected, the permeability decreases significantly as the GDL is compressed.

The dependence of permeability on porosity obtained for all samples tested are shown together in Fig. 5. The in-plane permeability of two samples (10BA and P75) differed distinctly when measured in two perpendicular directions. Other samples showed some tendency toward anisotropic behavior, but not to a significant extent (24BA and 34BA). The cloth material was only tested in the $0^{\circ}$ orientation due to the symmetry of the material when rotated $90^{\circ}$. Tests were performed on this material at $45^{\circ}$ and yielded results indistinguishable from those obtained at $0^{\circ}$. Only one experiment on the Toray 090 sample was performed due to limited material availability. Based on the random nature of this material, minimal anisotropy is expected. The permeability values for SGL 10BA are in the same range as those obtained by Ihonen et al. [12], although direct comparison 
is not possible since the thickness was not reported in this earlier study. Mathias et al. [3] reported the permeability of Toray 060 to be in the range of $5-10 \times 10^{-}$ $12 \mathrm{~m}^{2}$ when compressed to $75 \%$ of its original thickness which is in agreement with the value obtained for the structurally similar Toray 090 tested here. The solid and dashed lines in Fig. 5 correspond to the predictions of the CarmanKozeny model with the constants given in the legend. A detailed analysis of this and other permeability models is given in section 4.1 below.

The micrographs of the various materials in Fig. 6 show the variability of their pore structures. The SGL samples (10BA, 24BA and 34BA) all contained $5 \mathrm{wt} \%$ PTFE sintered into the pore structure, while the others contained no PTFE. The two samples that showed the most anisotropy in permeability (10BA and P75) also appear to have the most aligned fibers. The 10BA sample showed the most marked anisotropy in permeability with the higher value coinciding with the distinct "machine direction" shown in Fig. 6a. The 24BA and 34BA samples consisted of fibers randomly oriented in 2 dimensions and accordingly do not exhibit significant anisotropy in the plane. The Cloth ' $A$ ' material consists of woven bundles of fibers called 'tows'. The tightly bundled tows would presumably have a lower permeability than the overall assembled woven structure. 


\subsection{Inertial Coefficient}

Eq.(4) can be fit to the data for the variation of in-plane air flux with pressure drop to yield the inertial coefficient $\beta$. This coefficient is known to vary with permeability [16]. Liu et al. [18] developed the following correlation between $\beta$ and permeability from data collected from the literature:

$$
\beta=2.88 \times 10^{-6} \frac{\tau}{\varepsilon K}
$$

Fig. 7 shows the variation of inertial coefficient with permeability obtained experimentally for all materials and directions tested in the current study along with the correlation given in Eq.(7). For this analysis, the tortuosity of each sample was estimated using the Bruggeman equation [19]:

$$
\tau=\frac{1}{\varepsilon^{1 / 2}}
$$

With the exception of Toray 090 and E-Tek Cloth 'A', these results follow a similar trend as expected and described by the correlation of Liu et al. [18]. The deviation of Cloth 'A' from this trend may be expected due to its woven structure. The variation in the behaviour of Toray 090 is somewhat surprising given that its structure is similar to that of the other paper samples; however, this may be due to the fact that Toray 090 has a much more distinctly fiber-like web structure with no PTFE, binder or filler contained between the fibers. An analysis of the importance of inertial pressure losses occurring in the GDL during PEMFC operation is given in section 4.3 below. 


\subsection{Through-Plane Permeability}

The through-plane permeability of each material was also tested. Typical experimental results are given in Fig. 8 for two materials. Permeability was determined from the dependence of the air flux on pressure drop using Eq.(3) since the Forchheimer effect was not evident at the flow rates used in these experiments. The values of the through-plane permeability obtained in this way for all samples are presented in Table 2. The values reported are the average of three replicates. The average deviation of each replicate from the mean is also reported in Table 2. Comparison of these results with available literature values shows good agreement. Williams et al. [11] found the through-plane permeability for SGL 10BA and Toray 120 to be $31.0 \times 10^{-12} \mathrm{~m}^{2}$ and $8.69 \times 10^{-12} \mathrm{~m}^{2}$, respectively. Toray 120 is slightly thicker than the Toray 090 material used in the present study, but the permeability of the material should be similar given the similar structure. Ihonen et al. [12] reported a value of $18 \times 10^{-12} \mathrm{~m}^{2}$ for SGL 10BA in the through-plane direction. Ihonen et al. [12] found the in-plane permeability to be twice as high as the through-plane value which is in agreement with the present findings. Mathias et al. [3] tested the through-plane permeability of Toray 060 and obtained a range between 5 and $10 \times 10^{-12} \mathrm{~m}^{2}$. This is similar to the value they report for in-plane permeability of a compressed sample, indicating that the through-plane permeability is lower. 


\section{Discussion}

\subsection{Permeability}

The dependence of the permeability of a porous material on its porosity is often described by the Carman-Kozeny equation [20]:

$$
K=\frac{d_{f}^{2} \varepsilon^{3}}{16 k_{C K}(1-\varepsilon)^{2}}
$$

where $d_{f}$ is the fiber diameter, $\varepsilon$ is the porosity and $k_{\mathrm{CK}}$ is the Carman-Kozeny constant which depends on the type of media and is used as a fitting parameter. One of the goals of the present work is to provide an estimate of $k_{\mathrm{ck}}$ for the GDL materials tested.

As can be seen from the data in Fig. 5, the GDL permeability is well described by the Carman-Kozeny model with values of the Carman-Kozeny constants given in Table 3. The difference in the values of the constants is expected given the considerable differences in the fiber alignment and arrangement among the samples. Despite their structural differences, however, these materials still exhibit permeabilities with a common dependence on porosity that is well described by the Carman-Kozeny model.

A more comprehensive model for the permeability of porous fibrous materials has been developed by Tomadakis and Sotirchos [21-23]. A summary of this 
model and a comparison to a large volume of literature data has been compiled by Tomadakis and Robertson [20]. The Tomadakis - Sotirchos (TS) model enables the prediction of anisotropic permeability through 1D, 2D and 3D random fiber beds without employing any fitting parameters. The model requires only fiber diameter and porosity as input parameters. The TS model for absolute permeability is as follows:

$$
K=\frac{\varepsilon}{8(\ln \varepsilon)^{2}} \frac{\left(\varepsilon-\varepsilon_{p}\right)^{(\alpha+2)} d_{f}^{2}}{\left(1-\varepsilon_{p}\right)^{\alpha}\left[(\alpha+1) \varepsilon-\varepsilon_{p}\right]^{2}}
$$

where $\alpha$ and $\varepsilon_{p}$ are constants that depend on the fiber arrangement (aligned, random in $2 \mathrm{D}$ or $3 \mathrm{D}$ ) and on the direction of flow relative to the planes of the fibers. The values of $\alpha$ and $\varepsilon_{p}$ for the various possible scenarios are given in Table 4. This model is compared with the experimental results in Fig. 9. The data for Cloth 'A' were omitted from the comparison due to its woven structure. The data for the P75 sample were also excluded since it contains a considerable amount of non-fibrous solids (i.e. filler or binder) and so has a substantially lower permeability than the other materials. All samples are considered to have a $2 \mathrm{D}$ random fiber structure for the purposes of selecting parameters from Table 4 . The apparent fiber alignment in the 10BA sample suggests that the parameters for a 1D structure be appropriate (Fig. 6a). This approach predicted the permeability to flow normal to the fibers very well; however, the permeability to parallel flow was substantially overpredicted. On the other hand, the model could predict both directions reasonably well if a $2 \mathrm{D}$ structure was assumed and the 
parameters for parallel flow were applied to permeability in the direction of the fiber alignment (the $0^{\circ}$ direction) and parameters for normal flow to the permeability in the $90^{\circ}$ and through-plane directions. For the remaining samples, the parameters for normal flow were used to determine the through-plane permeability only and parallel flow parameters used for both in-plane directions. The results in Fig. 9 show excellent agreement between the experimental inplane permeability and those predicted by the TS model. Comparison of the through-plane permeability also shows quite favorable agreement. For instance, the through-plane permeability of Toray 090 is estimated by Eq.(10) using parameters corresponding to flow normal to the fibers to be $9.75 \times 10^{-12} \mathrm{~m}^{2}$, which compares well with the experimental value of $8.99 \times 10^{-12} \mathrm{~m}^{2}$.

\subsection{Tortuosity}

In addition to predicting the permeability as a function of porosity, the TS model provides a means of estimating tortuosity which is used to calculate the effective diffusivity as follows:

$$
D_{e f f}=\frac{\varepsilon}{\tau} D_{A B}
$$

where $D_{\mathrm{AB}}$ is the molecular diffusivity. The ability to predict the change in effective diffusivity in a compressed GDL would be useful since many recent fuel cell models include the under-land area in the modeling domain [24,25]. Even a 
fuel cell model that does not consider convection in the GDL requires this information. The TS model expression for tortuosity is as follows:

$$
\tau=\left(\frac{1-\varepsilon_{p}}{\varepsilon-\varepsilon_{p}}\right)^{\alpha}
$$

where $\alpha$ and $\varepsilon_{p}$ are the same constants used for estimating the permeability. Tortuosity is more commonly predicted using the Bruggeman equation [26] given in Eq.(8). A possible benefit of using Eq.(12) rather than the Bruggeman equation is that the effect of anisotropy can be included in estimating the effective diffusivity. Using the 2D parameters from Table 4 , the values determined from Eq.(12) agree within $20 \%$ of those obtained using Eq.(8) for porosities expected in a fuel cell $(\varepsilon>0.6)$. More interestingly, Eq. (12) predicts that the tortuosity will vary by as much as $15 \%$ between the in-plane and through-plane directions. Unfortunately, no experimental evidence is available to validate these predictions for GDL materials. Nam and Kaviany [27] have investigated these and other tortuosity estimates using a pore network model of a GDL structure and found the TS model to best describe their numerical results.

\subsection{Importance of Inertial Losses in GDLs}

The Darcy equation (Eq.(3)) is a special case of the more general Forchheimer equation (Eq.(4)) and is only applicable for creeping flow rates through porous media. When the flow rate is higher, inertial losses become significant and Darcy's law does not accurately describe pressure drops. Zeng and Grigg [28] 
have recently discussed the problem of determining the point at which inertial effects become significant. To assess this quantitatively, they defined a dimensionless Forchheimer number $(F o)$ as the ratio of the inertial pressure loss contribution to the viscous pressure drop contributions:

$$
F_{O}=\frac{K \beta \rho v}{\mu}
$$

This definition of $F o$ is equivalent to the Reynolds number where $K \beta$ is the characteristic length [16]. A higher value of Fo signifies that inertial effects are more important and that the use of Darcy's law to calculate pressure drops becomes increasingly inaccurate. The amount of error incurred by neglecting inertial effects can be calculated as follows:

$$
E=\frac{F o}{1+F o}
$$

In their work, Zeng and Grigg suggested that an error of $10 \%$ is tolerable for most engineering calculations, which sets the critical Fo number at 0.11 . An error tolerance of $10 \%$ is arbitrary, and some applications may require higher accuracy. It is worthwhile to determine Fo values for conditions typically prevailing during PEMFC operation to determine whether inertial effects need to be considered. The data of Williams et al. [4] provide an excellent test case for this calculation since these showed that convection through the GDL was significant in their work. In their experiments, Williams et al. varied the inlet flow rate of air to the cathode between 0.050 and 0.500 SLPM. Based on the 
description of the experimental conditions, the mass flow rate of heated and humidified air into the cell is estimated to vary between 1.5 and $15 \times 10^{-6} \mathrm{~kg} / \mathrm{s}$, which corresponds to a mass flux along the single serpentine flow channel of 2.2 to $22 \mathrm{~kg} / \mathrm{m}^{2}$.s and a channel Reynolds number ranging from 110 to 1100 . It is impossible to know precisely how much of this flow bypassed the channel and flowed through the GDL. Nonetheless, the modeling of Pharaoh [5] suggests that about $10 \%$ of the flow bypasses through the GDL when the channel $R e$ is 100 for a similar geometry and GDL permeability. Using this estimate with the Williams et al. [4] data for an inlet flow of 0.05 SLPM (i.e. Rechannel $=110$ ), the mass flux through the GDL is about $0.025 \mathrm{~kg} / \mathrm{m}^{2} \cdot \mathrm{s}$, where the flow area is based on a channel length of $0.025 \mathrm{~m}$ and a GDL thickness of $250 \mu \mathrm{m}$. In order to calculate $F_{O}$, an estimate of the transport properties existing in the experiments of Williams et al. [4] is required. To do this, parameters of Toray 090 given in Table 1 and Table 3 were used (i.e. $d_{\mathrm{f}}, t_{\mathrm{o}}, \varepsilon_{0}$ and $k_{\mathrm{ck}}$ ). Compressed porosity was estimated by inserting a compressed thickness of $250 \mu \mathrm{m}$ into Eq.(6). Compressed porosity was used to calculate both permeability using Eq. (9) and tortuosity using Eq.(8). The inertial coefficient was not calculated using Eq.(7) since Toray 090 deviated significantly from the correlation. Instead, the experimentally measured $\beta$ of $8.22 \times 10^{4} \mathrm{~m}^{2}$ for $t_{\mathrm{c}}=250 \mu \mathrm{m}$ was used. The resulting Fo value corresponding to this mass flux is only 0.0013 . However, if instead it is assumed that $50 \%$ of the gas bypasses through the GDL with the highest flow rate used by Williams et al. [4] $(0.5$ SLPM, Rechannel $=1100)$ the Fo number becomes 0.055 , indicating an error of $5.2 \%$ is incurred by use of Darcy's 
law. This error is not negligible and the Fo number could increase to even higher values under different circumstances, such as the use of a larger cell or higher inlet humidity. 


\section{Conclusions}

The gas permeability of several common GDL materials was measured in three perpendicular directions. In-plane measurements were made as a function of compressed GDL thickness. Not only does the demonstrated method enable the simulation of in-situ cell conditions where considerable GDL compression exists, but it also provided a means of varying the porosity of the sample. The data were well described by the Carman-Kozeny model which predicts permeability as a function of porosity. Carman-Kozeny constants were determined for each material for both in-plane directions. The data were also compared to the permeability model of Tomadakis and Sotirchos [20] and found to agree well. This predictive model requires no fitting parameters and can be applied to anisotropic materials. Through-plane and in-plane permeabilities were both well predicted. An added benefit of this model is that it also allows estimates of the tortuosity and effective diffusivity to be made that account for the anisotropy of the material.

An analysis of the non-Darcy effect in GDLs was also undertaken. The inertial coefficients for all materials tested were found to be inversely proportional to the permeability and were well described by the correlation of Liu et al. [18]. Based on these findings, the importance of inertial effects in the flow through the GDL in an operating fuel cell was investigated. For typical fuel cell conditions, the inplane flow through the GDL is low enough that Darcy's law is accurate to better than $5 \%$. Although this error is low, it is not negligible and could increase to a 
significant amount if flow conditions change. Therefore, it would be prudent to check the validity of Darcy's law on a case-by-case basis.

This work should prove useful to future modeling studies that aim to describe 3D effects in PEMFCs since the determination of permeabilities in the three directions described here will allow the formulation of a permeability tensor. Also, an estimate for the effective diffusivity tensor can be made based on these findings. The detailed investigation of the effect of GDL compression on permeability in this study will also be valuable for further improving the assembly of PEMFC stacks. 


\section{Acknowledgements}

The authors acknowledge the financial support of the Natural Sciences and Engineering Research Council of Canada (NSERC) during the course of this project. They also wish to thank SGL Carbon and Ballard Power Systems for donating GDL sample materials. 


\section{Nomenclature}

\begin{tabular}{|c|c|c|}
\hline Symbol & Description & Units \\
\hline$\alpha$ & Numerical Constant in Eq.(10)and Eq.(12) & --- \\
\hline$\beta$ & Inertial Coefficient & $m^{-1}$ \\
\hline$d_{f}$ & Fiber Diameter & $\mathrm{m}$ \\
\hline$\varepsilon$ & Porosity & --- \\
\hline$\varepsilon_{p}$ & Numerical Constant in Eq.(10)and Eq. (12) & --- \\
\hline Fo & Forchheimer Number & --- \\
\hline kck & Carman-Kozeny Constant & --- \\
\hline K & Permeability Coefficient & $\mathrm{m}^{2}$ \\
\hline L & Length of Test Specimen & $\mathrm{m}$ \\
\hline $\mathrm{m}^{\prime}$ & Mass Flux & $\mathrm{kg} / \mathrm{m}^{2} \cdot \mathrm{s}$ \\
\hline MW & Molecular Weight & $\mathrm{kg} \cdot \mathrm{mol}^{-1}$ \\
\hline$\mu$ & Kinematic Viscosity & $\mathrm{Pa} \cdot \mathrm{s}$ \\
\hline$P$ & Pressure & $\mathrm{Pa}$ \\
\hline $\mathrm{R}$ & Universal Gas Constant & $\mathrm{J} \cdot \mathrm{mol}^{-1}$ \\
\hline $\operatorname{Re}$ & Reynolds Number & --- \\
\hline$\rho$ & Density & $\mathrm{kg} \cdot \mathrm{m}^{-3}$ \\
\hline $\mathrm{T}$ & Temperature & $\mathrm{K}$ \\
\hline$\tau$ & Tortuosity & --- \\
\hline v & Superficial Velocity & $\mathrm{m} \cdot \mathrm{s}^{-1}$ \\
\hline$V_{p}$ & Pore Volume & $\mathrm{m}^{3}$ \\
\hline
\end{tabular}


$\begin{array}{lll}V_{b} & \text { Bulk Volume } & \mathrm{m}^{3}\end{array}$

$\begin{array}{lll}\text { Vs } & \text { Solid Volume } & \mathrm{m}^{3}\end{array}$

Subscripts

C Compressed

o Uncompressed 


\section{References}

1. US DOE, Fuel cell technologies roadmap. 08-10-05a. 8-5-2005.

2. F. A. L. Dullien, Porous Media: Fluid Transport and Pore Structure, Academic Press, New York, 1992.

3. M. F. Mathias, J. Roth, J. Fleming, and W. Lehnert, "Diffusion media materials and characterization," Handbook of Fuel Cells - Fundamentals, Technology and Applications. Volume 3, Part 1., W. Vielstich, H. A. Gasteiger, and A. Lamm, eds., (John Wily \& Sons, New York, 2003), 517537.

4. M. V. Williams, H. R. Kunz, and J. M. Fenton, Influence of convection through gas-diffusion layers on limiting current in PEM FCs using a serpentine flow field, J Electrochem Soc 151 (2004) A1617-A1627.

5. J. G. Pharoah, On the permeability of gas diffusion media used in PEM fuel cells, J Power Sources 144 (2005) 77-82.

6. P. H. Oosthuizen, L. Sun, and K. B. McAuley, The effect of channel-tochannel gas crossover on the pressure and temperature distribution in PEM fuel cell flow plates, App Therm Eng 25 (2005) 1083-1096.

7. W. Sun, B. A. Peppley, and K. Karan, Modeling the Influence of GDL and flow-field plate parameters on the reaction distribution in the PEMFC cathode catalyst layer, J Power Sources 144 (2005) 42-53.

8. T. V. Nguyen, Gas distributor design for proton-exchange-membrane fuel cells, J Electrochem Soc 143 (1996) 103-105. 
9. U. Pasaogullari and C. Y. Wang, Two-phase transport and the role of micro-porous layer in polymer electrolyte fuel cells, Electrochim Acta 49 (2004) 4359-4369.

10. W. Lee, C.-H. Ho, J. W. Van Zee, and M. Murthy, The effects of compression and gas diffusion layers on the performance of a PEM fuel cell, J Power Sources 84 (1999) 45-51.

11. M. V. Williams, E. Begg, L. Bonville, H. R. Kunz, and J. M. Fenton, Characterization of gas diffusion layers for PEMFC, J Electrochem Soc 151 (2004) 1173-1180.

12. J. Ihonen, M. Mikkola, and G. Lindbergh, Flooding of gas diffusion backing in PEFCs: Physical and electrochemical characterization, J Electrochem Soc 151 (2004) 1152-1161.

13. Mueller, B, Zawodzinski, T. A., Bauman, J, Uribe, F, and Gottesfeld, S, Carbon cloth diffusion backings for high performance PEFC cathodes, Proceedings of the Second International Symposium on Proton Conducting Membrane Fuel Cells,Fuller, T. F. and Gottesfeld, S, Eds. 9827 (1999) pp. 1-9.

14. M. Prasanna, H. Y. Ha, E. A. Cho, S. A. Hong, and I. H. Oh, Influence of cathode gas diffusion media on the performance of the PEMFCs, J Power Sources 131 (2004) 147-154.

15. H. Dohle, R. Jung, N. Kimiaie, J. Mergel, and M. Muller, Interaction between the diffusion layer and the flow field of polymer electrolyte fuel 
cells - Experiments and simulation studies, J Power Sources 124 (2003) $371-384$.

16. J. Geertsma, Estimating the coefficient of inertial resistance in fluid flow through porous media, Soc Pet Eng J 10 (1974) 445-450.

17. R. B. Bird, W. E. Stewart, and E. N. Lightfoot, Transport Phenomena, John Wiley \& Sons, New York, 1960.

18. X. Liu, F. Civan, and R. D. Evans, Correlation of the non-Darcy flow coefficient, J Can Petro Tech 34 (1995) 50-54.

19. J. van Brakel and P. M. Heertjes, Analysis of diffusion in macroporous media in terms of a porosity, a tortuosity and a constrictivity factor, Int $\mathrm{J}$ Heat Mass Trans 17 (1974) 1093-1103.

20. M. M. Tomadakis and T. J. Robertson, Viscous permeability of random fiber structures: Comparison of electrical and diffusional estimates with experimental and analytical results, J Composite Materials 39 (2005) 163188.

21. M. M. Tomadakis and S. V. Sotirchos, Ordinary and transition regime diffusion in random fiber structures, AICHE J 39 (1993) 397-412.

22. M. M. Tomadakis and S. V. Sotirchos, Effective diffusivities and conductivities of random dispersions of nonoverlapping and partially overlapping unidirectional fibers, J Chem Phys 99 (1993) 9820.

23. M. M. Tomadakis and S. V. Sotirchos, Transport properties of random arrays of freely overlapping cylinders with various orientation distributions, J Chem Phys 98 (1993) 616. 
24. R. Roshandel, B. Farhanieh, and E. Saievar-Iranizad, The effects of porosity distribution variation on PEM fuel cell performance, Renewable Energy 30 (2005) 1557-1572.

25. G. Lin and T. V. Nguyen, A two-dimensional two-phase model of a PEM fuel cell, J Electrochem Soc 153 (2006) A372-A382.

26. A. Z. Weber and J. Newman, Modeling transport in polymer-electrolyte fuel cells, Chem Rev 104 (2004) 4679-4726.

27. J. H. Nam and M. Kaviany, Effective diffusivity and water-saturation distribution in single- and two-layer PEMFC diffusion medium, Int $\mathrm{J}$ Heat Mass Trans 46 (2003) 4595-4611.

28. Z. Zeng and R. Grigg, A criterion for non-darcy flow in porous media, Trans Porous Media 63 (2006) 57-69. 


\section{Figures}

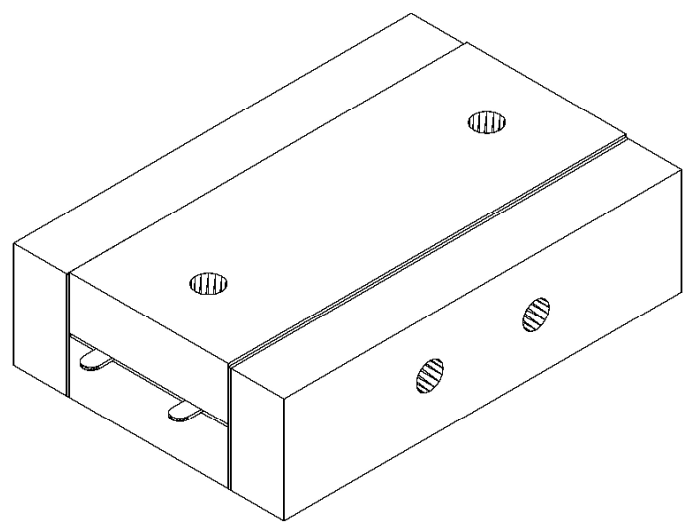

(a)

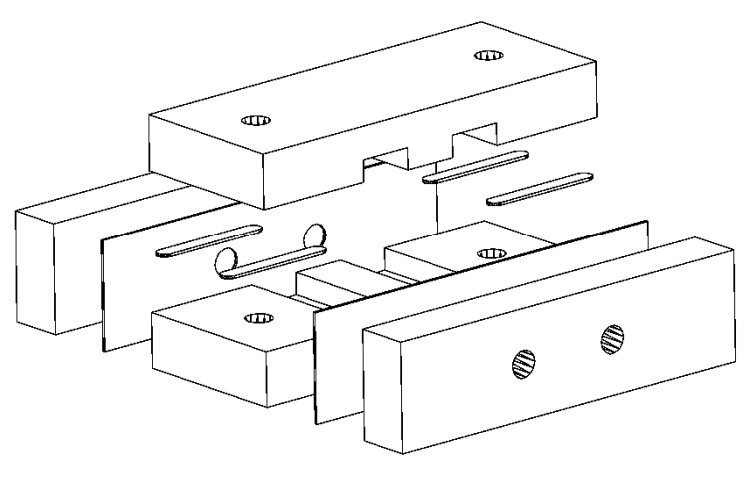

(b)

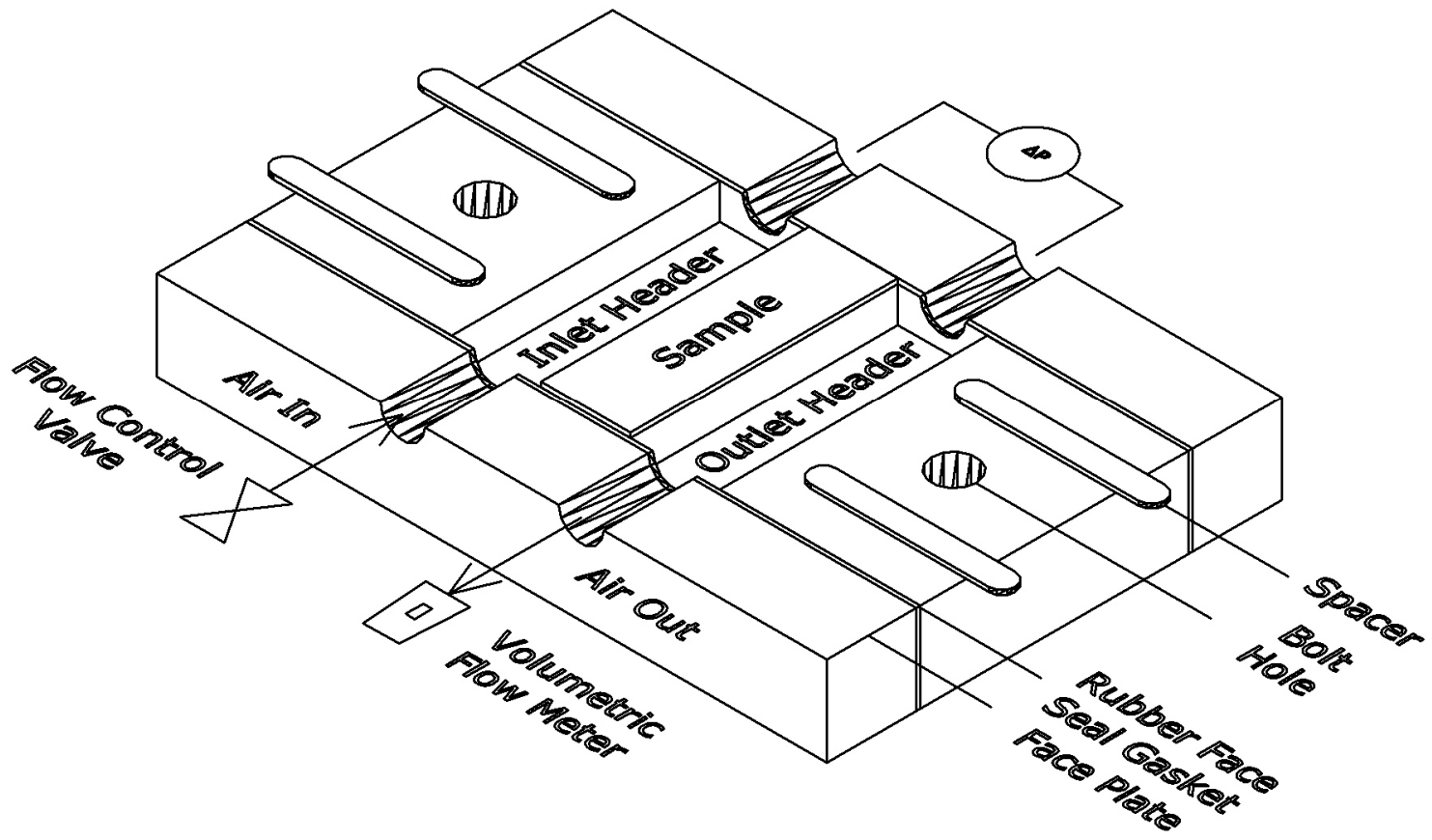

(c)

Fig. 1. Experimental apparatus for in-plane permeability measurement. (a) assembled view (b) exploded view (c) sectioned view to show internal components. 


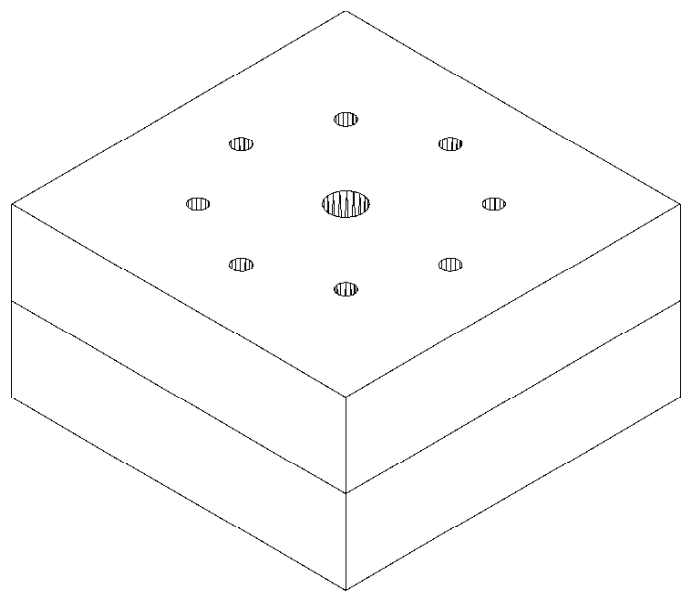

(a)

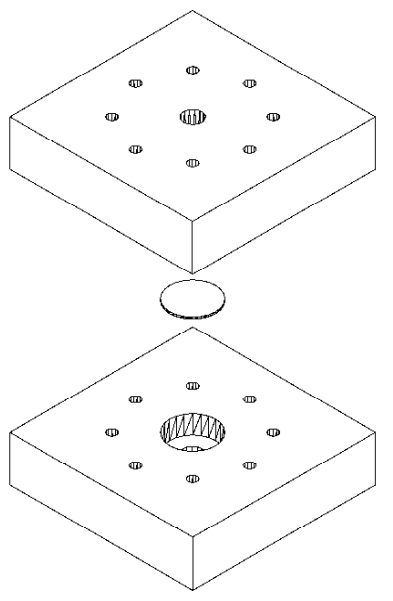

(b)

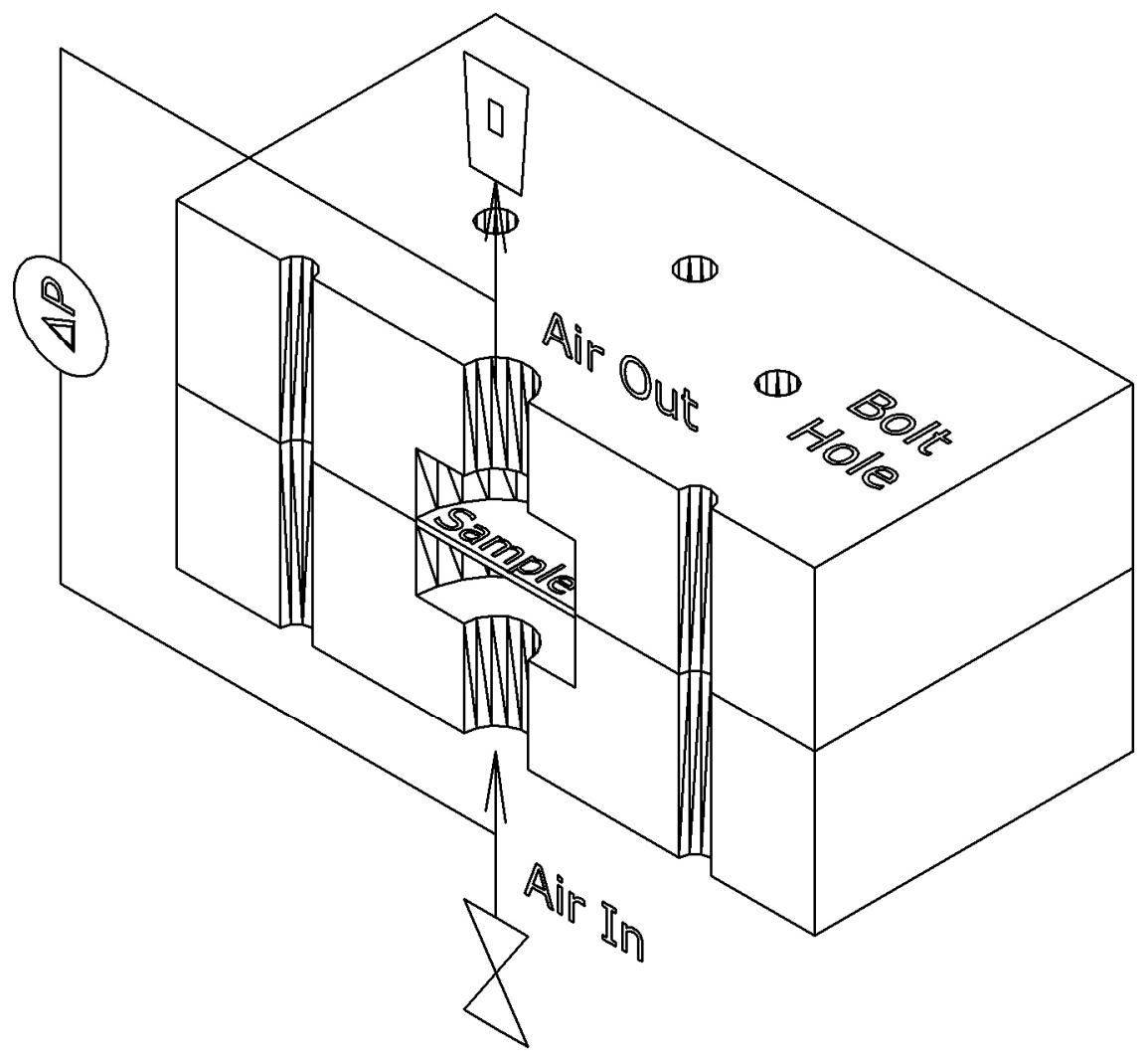

(c)

Fig. 2. Experimental apparatus for through-plane permeability measurement. (a) assembled view (b) exploded view (c) sectioned view to show internal components. 


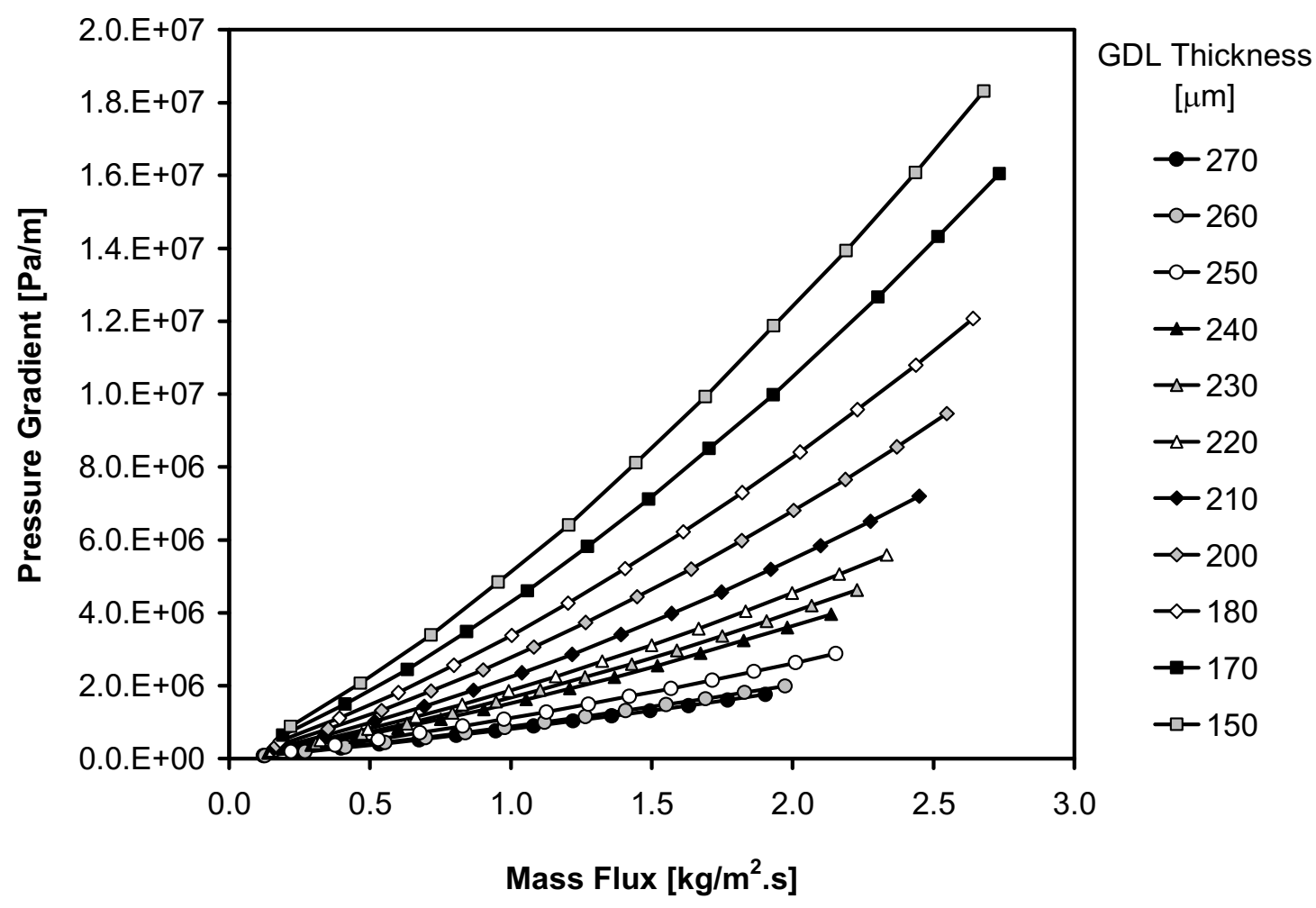

Fig. 3. Experimental data of in-plane pressure drop as a function of air flux for SGL 34BA material oriented in the $0^{\circ}$ direction and compressed to different thicknesses. 

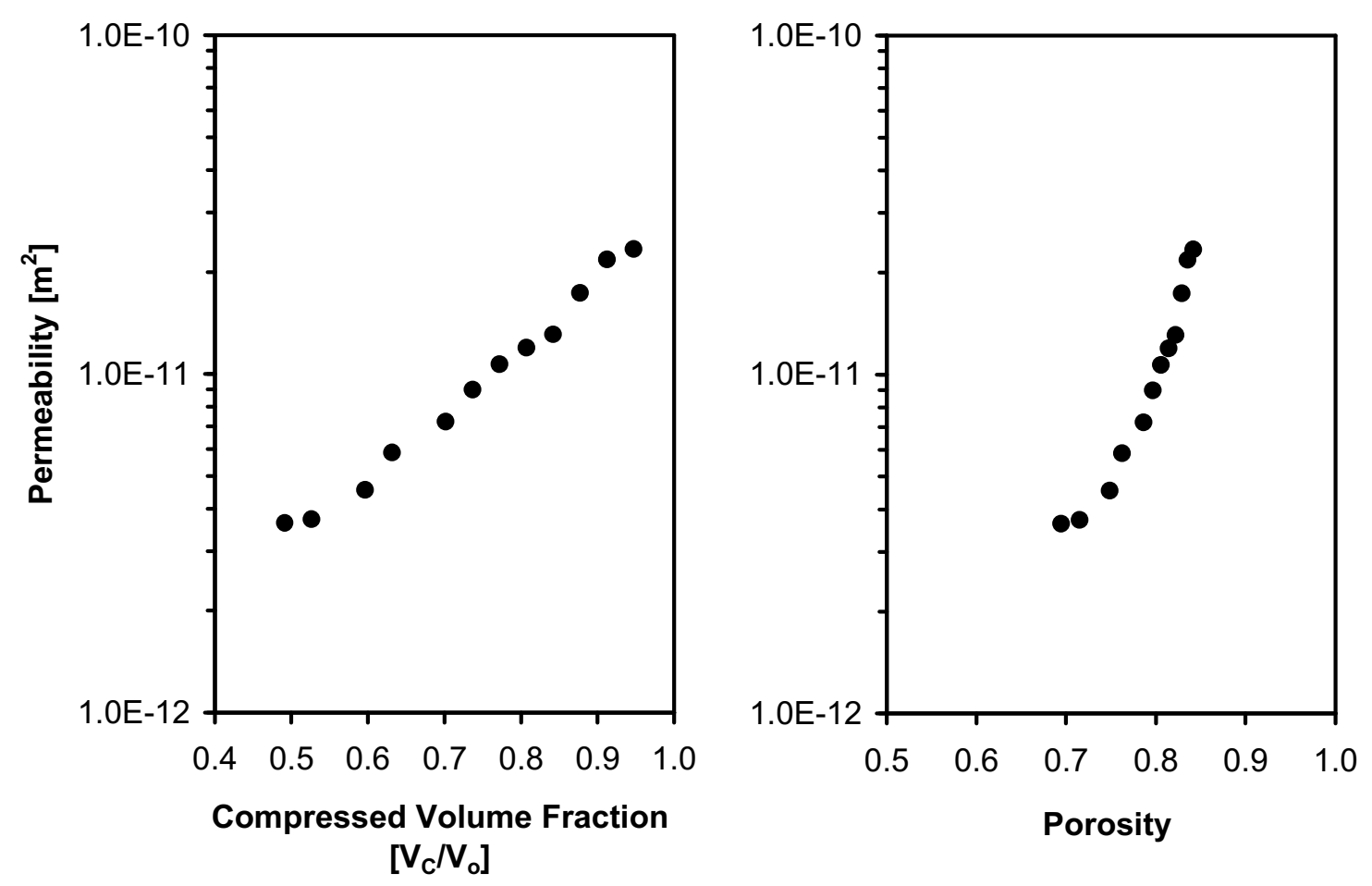

Fig. 4. Variation of (a) permeability with sample compressed volume fraction and (b) permeability with sample porosity converted using Eq.(6) for SGL 34BA GDL sample oriented in the $0^{\circ}$ direction. 

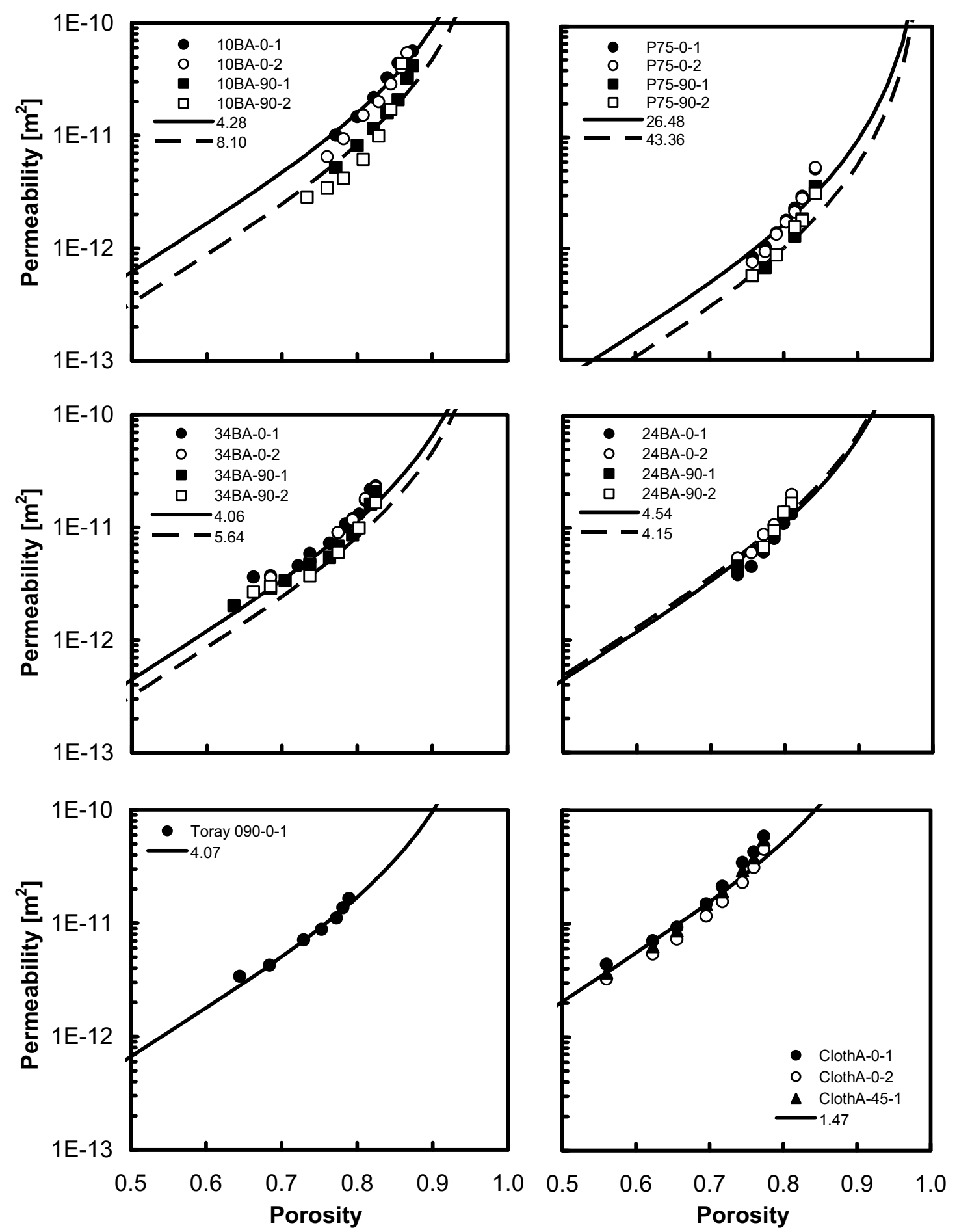

Fig. 5. Variation of in-plane permeability with porosity for all samples tested. Solid and dashed lines show the Carman-Kozeny model with the constant given 
in the legend. Sample names are given in the legend as 'material'-'direction''replicate'.

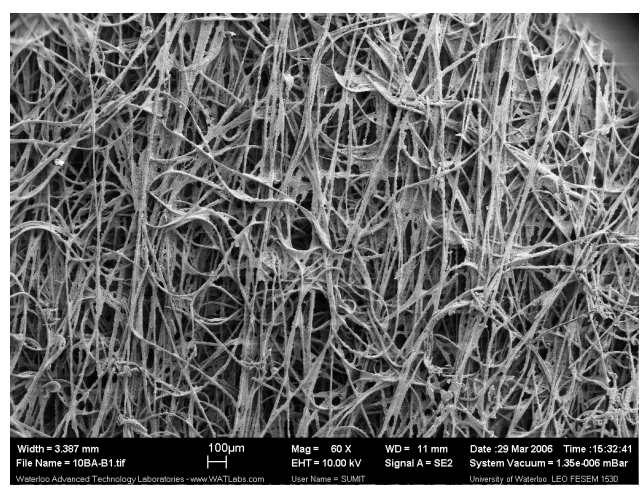

(a)

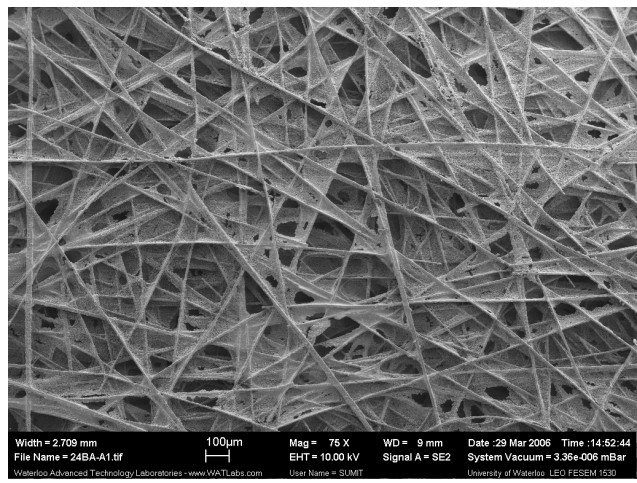

(c)

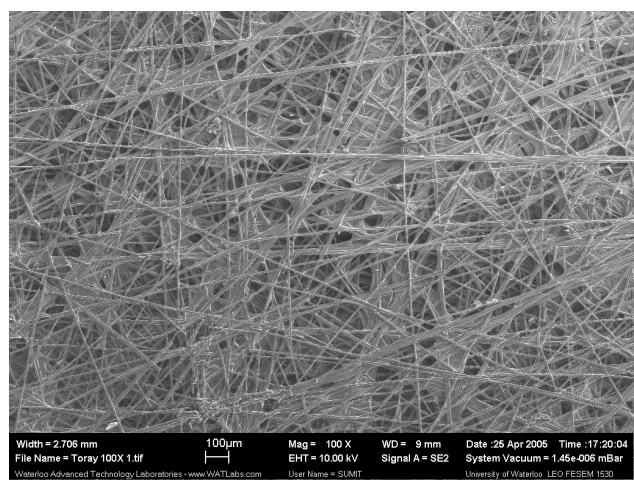

(e)

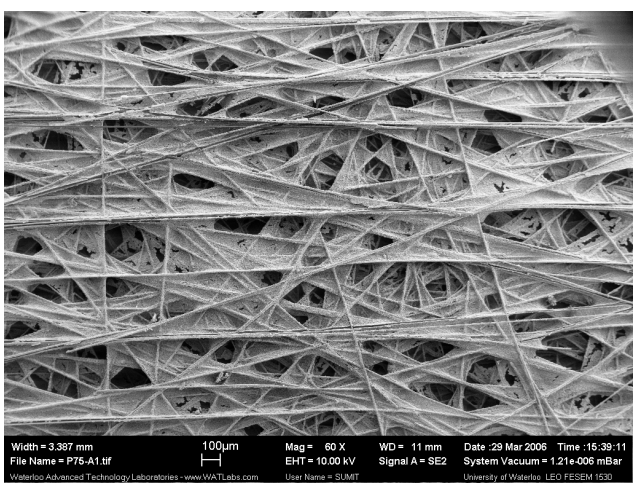

(b)

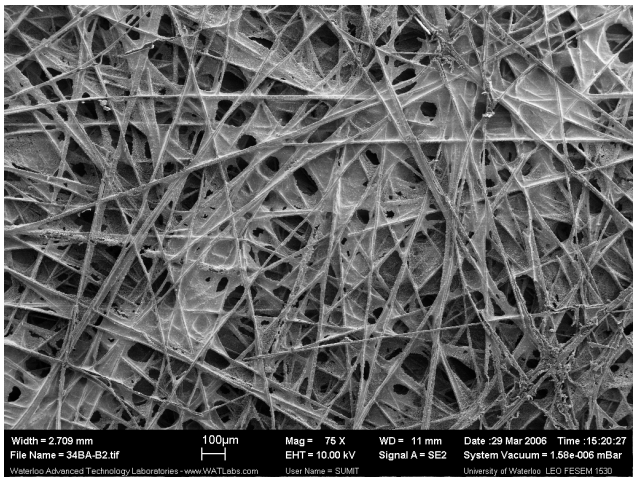

(d)

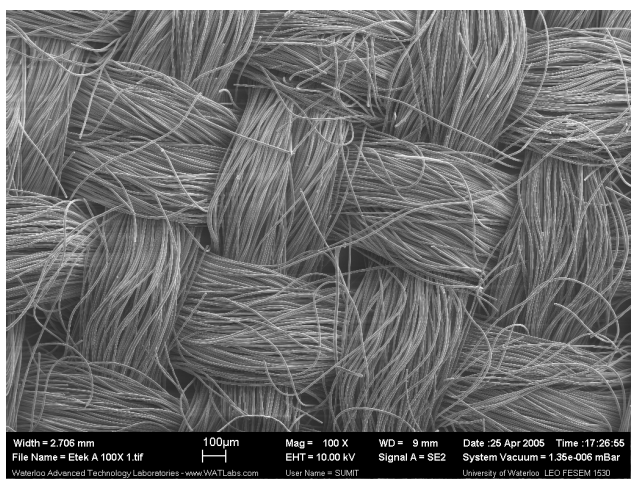

(f)

Fig. 6. Micrographs of the materials used in this study. (a) SGL 10BA (b) Ballard P75 (c) SGL 24BA (d) SGL 34BA (e) Toray 090 (f) E-Tek Cloth 'A'. Images (a) 
and (b) show an area of $350 \times 240 \mu \mathrm{m}$ and the remaining images show an area of $300 \times 200 \mu \mathrm{m}$.

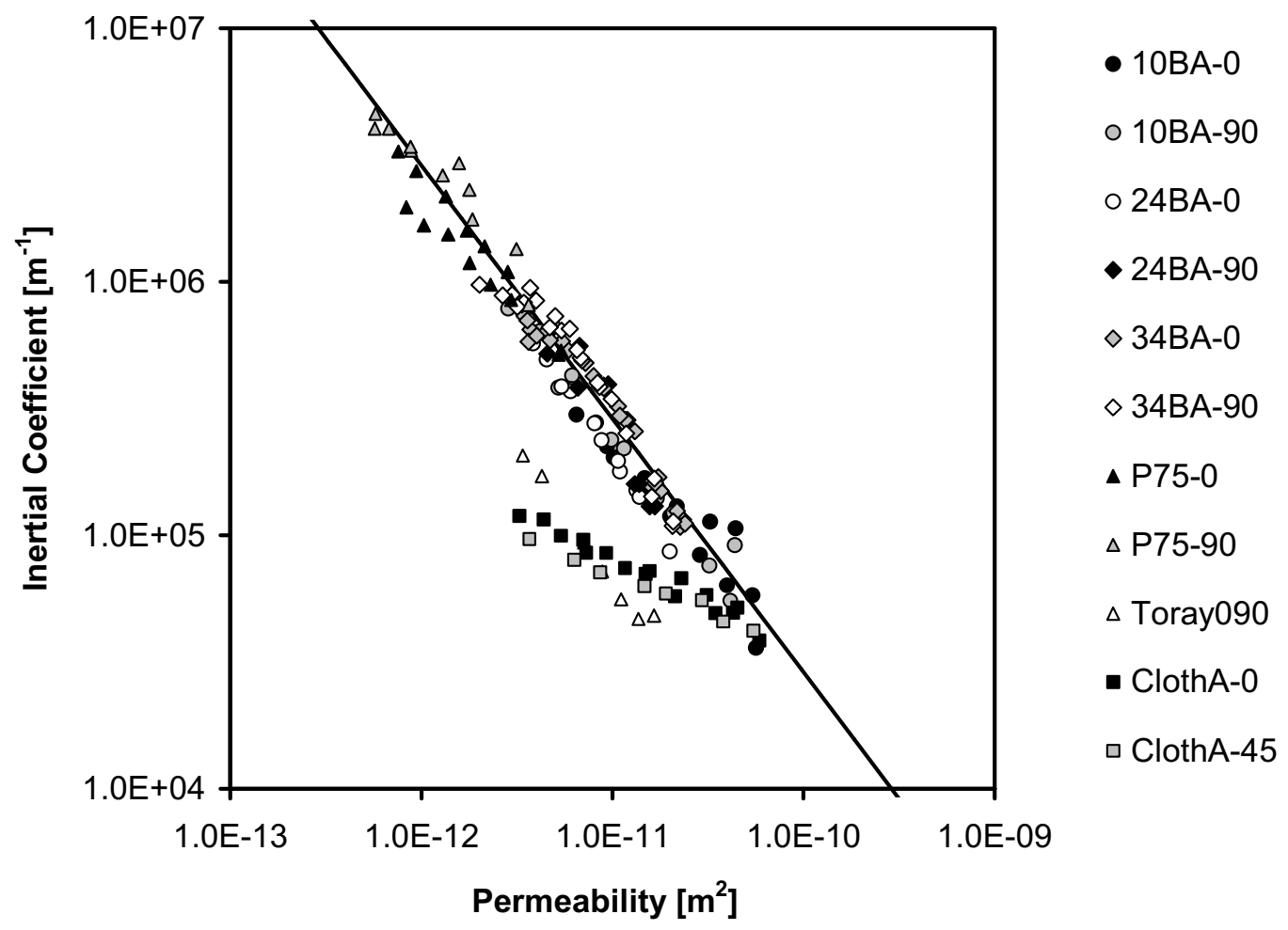

Fig. 7. Variation of inertial coefficient with permeability for all materials tested. Solid line represents the correlation of Liu et al. [18] 


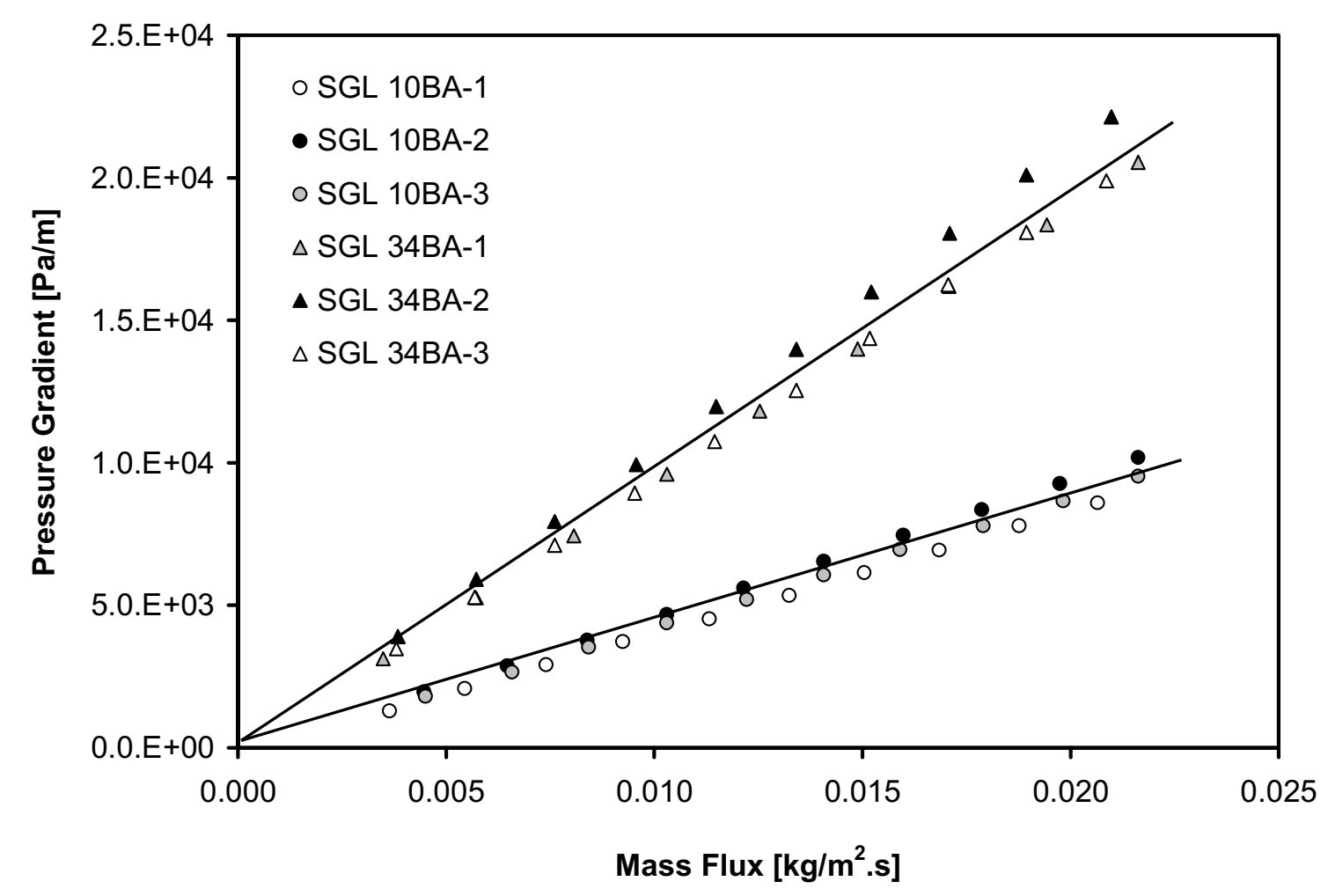

Fig. 8. Experimental data of through-plane pressure drop as a function of air flux for SGL 34BA (triangles) and SGL 10BA (circles). Replicates were performed on 3 separate samples, each taken from the same sheet of material. 

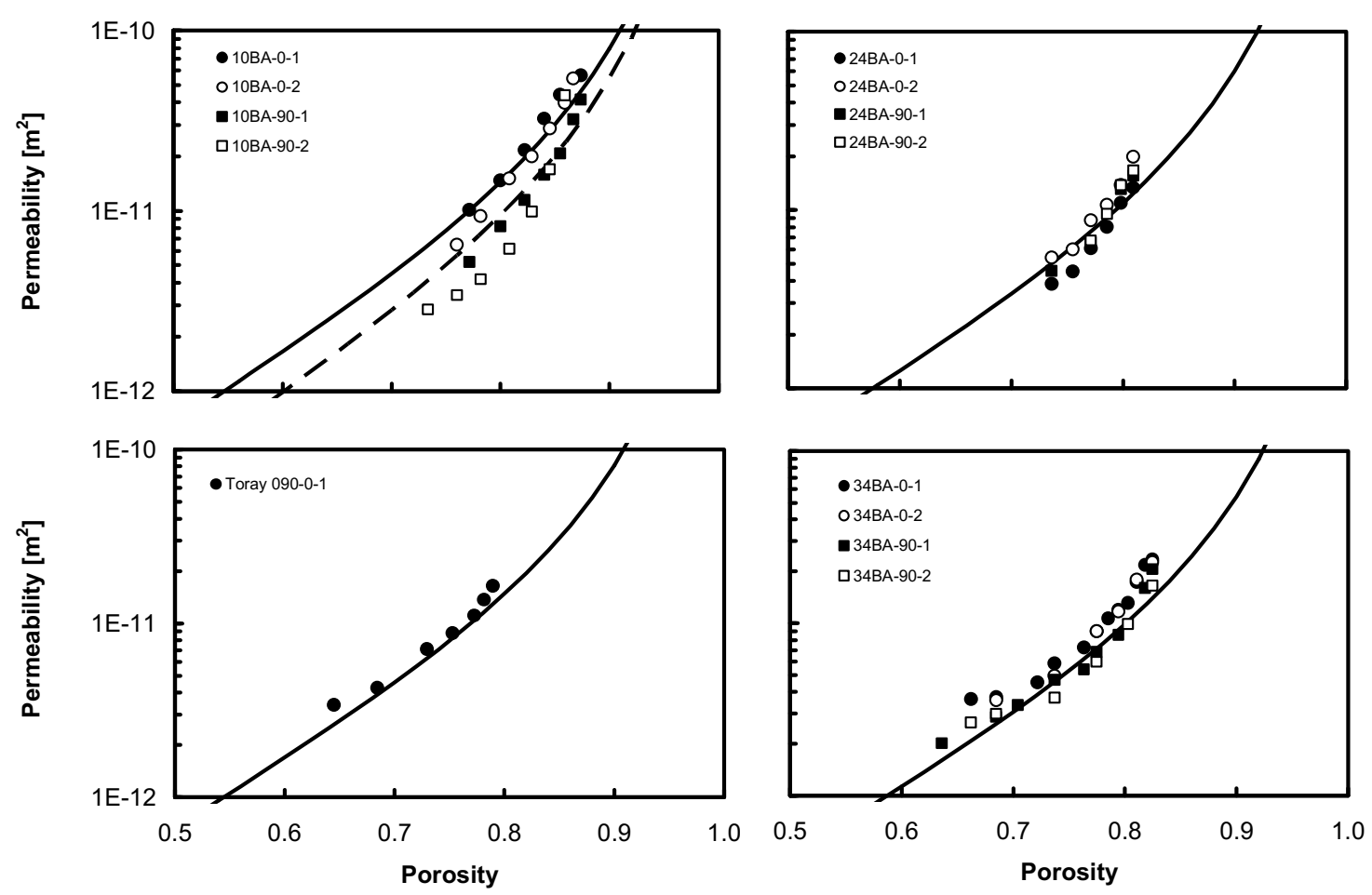

Fig. 9. Comparison of experimental results to the predictions of the TS model, Eq.(10). The solid lines were calculated using parameters for 2D parallel flow. The dashed line was calculated using parameters for 2D normal flow. 


\section{Tables}

Table 1. GDL materials and measured properties used in this study.

$\begin{array}{ccccc}\text { Material } & \begin{array}{c}\text { Porosity } \\ {[\mathrm{mL} / \mathrm{mL}]}\end{array} & \begin{array}{c}\text { Thickness } \\ {[\mu \mathrm{m}]}\end{array} & \begin{array}{c}\text { Fiber Diameter } \\ {[\mu \mathrm{m}]}\end{array} & \begin{array}{c}\text { PTFE Loading } \\ {[\mathrm{wt} . \%]}\end{array} \\ \text { SGL 10BA } & 0.88 & 400 & 9.2+/-1.4 & 5 \\ \text { SGL 24BA } & 0.81 & 195 & 8.0+/-0.69 & 5 \\ \text { SGL 34BA } & 0.84 & 285 & 7.6+/-0.93 & 5 \\ \text { Ballard P75 } & 0.85 & 210 & 7.4+/-0.35 & 0 \\ \text { Toray 090 } & 0.80 & 290 & 9.2+/-0.78 & 0 \\ \text { E-Tek Cloth 'A' } & 0.78 & 360 & 9.9+/-0.67 & 0\end{array}$

Table 2. Through-plane permeability values of each sample.

$\begin{array}{ccc}\text { Material } & \begin{array}{c}\mathrm{K}_{z} \\ {\left[\mathrm{~m}^{2}\right]}\end{array} & \begin{array}{c}\text { Average } \\ \text { Deviation [\%] }\end{array} \\ \text { SGL 10BA } & 37.4 \times 10^{-12} & 3.76 \\ \text { SGL 24BA } & 14.5 \times 10^{-12} & 7.02 \\ \text { SGL 34BA } & 16.3 \times 10^{-12} & 5.05 \\ \text { Ballard P75 } & 5.70 \times 10^{-12} & 5.96 \\ \text { Toray 090 } & 8.99 \times 10^{-12} & 1.01 \\ \text { E-Tek Cloth 'A' } & 69.4 \times 10^{-12} & 5.26\end{array}$

Table 3. Carman-Kozeny constants determined for each material and direction.

$\begin{array}{ccccc}\text { Material } & \mathrm{k}_{\mathrm{ck}, \mathrm{x}} & \begin{array}{c}\text { Average } \\ \text { Error [\%] }\end{array} & \mathrm{k}_{\mathrm{ck}, \mathrm{y}} & \begin{array}{c}\text { Average } \\ \text { Error [\%] }\end{array} \\ \text { SGL 10BA } & 4.28 & 15.2 & 8.10 & 25.0 \\ \text { SGL 24BA } & 4.54 & 17.5 & 4.2 & 15.1 \\ \text { SGL 34BA } & 4.06 & 16.1 & 5.6 & 21.1\end{array}$




$\begin{array}{ccccc}\text { Ballard P75 } & 26.5 & 16.7 & 43.5 & 14.0 \\ \text { Toray 090 } & 4.07 & 6.3 & --- & --- \\ \text { E-Tek Cloth 'A' } & 1.446 & 17.3 & --- & ---\end{array}$

Table 4. Constants used in the TS model, Eq.(10).

$\begin{array}{cccc}\text { Structure } & \text { Flow Direction } & \varepsilon p & \alpha \\ \text { 1D } & \text { Parallel } & 0 & 0 \\ & \text { Normal } & 0.33 & 0.707 \\ \text { 2D } & \text { Parallel } & 0.11 & 0.521 \\ & \text { Normal } & 0.11 & 0.785 \\ \text { 3D } & \text { All Directions } & 0.037 & 0.661\end{array}$

